\title{
COMMENT
}

Check for updates

\section{Electromagnetic counterparts of gravitational wave sources at the Very Large Telescope}

\author{
Andrew J. Levan $\mathbb{(}^{1,2 \bowtie}$ and Peter G. Jonker $\mathbb{1}^{1,3 凶}$
}

Andrew J. Levan and Peter G. Jonker discuss, on behalf of the Electromagnetic counterparts of gravitational wave sources at the Very Large Telescope (ENGRAVE), how the collaboration was formed and what its goals are in the era of multi-messenger astronomy.

Astronomy has always relied on measuring the properties of photons using an array of increasingly sophisticated instrumentation. In the twentieth century, our understanding of the Universe was dramatically expanded by multi-wavelength astronomy, which enabled spectacular discoveries, from pulsars and $\gamma$-ray bursts to the cosmic microwave background. But with the birth of multi-messenger astronomy, which exploits non-photonic carriers of information, entirely new windows into the extreme and violent Universe have opened ${ }^{1}$.

\section{The first event}

For many astronomers, the period around 17 August 2017 will be remembered as one of the most remarkable of their careers. The prospect of observing the merger of two neutron stars with gravitational waves and in electromagnetic radiation had been a tantalizing one. Would a burst of $\gamma$-rays be visible or a radio flare, or perhaps a faint, fast explosion powered by the synthesis of some of the heaviest elements in nature? Within the course of a few days, the answer to these questions became clear: all of these signatures were visible. The observations of the neutron star merger of 17 August 2017, named GW170817, provided clues to many other problems, from the origin of short $\gamma$-ray bursts to the location of the heavy element production and the expansion rate of the Universe. Seldom has a single astronomical event yielded so much insight, and equally seldom has one been observed with the amount of resources committed to GW 170817.

Crucial to this campaign were the facilities of the European Southern Observatory (ESO). Its four Very Large Telescopes (VLTs) located in northern Chile, each equipped with specialized instruments, create a unique and powerful tool, which can be used to simultaneously take images in optical and near-infrared light, disperse the light into spectra and measure its polarization state, a set of observations impossible from any other single observatory (see FIG. 1, which illustrates how the VLT instruments were able to simultaneously observe the GW170817 event). For example, the collection of spectra obtained from ESO's X-shooter spectrograph simultaneously span the ultraviolet to the infrared, providing the most constraining spectra available for GW170817 $\left(\mathrm{REFS}^{2,3}\right)$, paired with simultaneous polarimetry to probe the geometry of the event $t^{4}$. As the source faded, using multiple telescopes to get deep observations across the optical and the infrared spectral windows enabled meaningful constraints even as the electromagnetic counterpart reached the limits of observability.

\section{The aftermath}

During August and September 2017 more than 5,000 images and spectra of GW170817 were taken with 14 instruments on seven different ESO telescopes. Strikingly, they were taken from around ten different programmes, each with different principal investigators and slightly different instrumental set-ups. Although many of these programmes subsequently combined forces for analysis, the observations were not as well coordinated as they might have been. They did not minimize duplication, cross-calibration of slightly different set-ups became challenging and on occasion the most urgent data were not obtained; instead data of lower constraining power were taken. Although the project was a huge success it was also clear that there was room for future improvement, especially in an era when many more multi-messenger events are to be discovered and the repetition of such an intense campaign would not be plausible.

\section{A new collaboration}

To prepare for future observations, ESO hosted an open meeting in February 2018. The participants agreed near-unanimously that better coordination would lead to better science. A series of follow-up meetings determined the core underlying principles and goals: optimize for the best scientific output, while maintaining flexibility. A second important goal is to allow visibility for researchers at earlier career stages. These ideas led to 


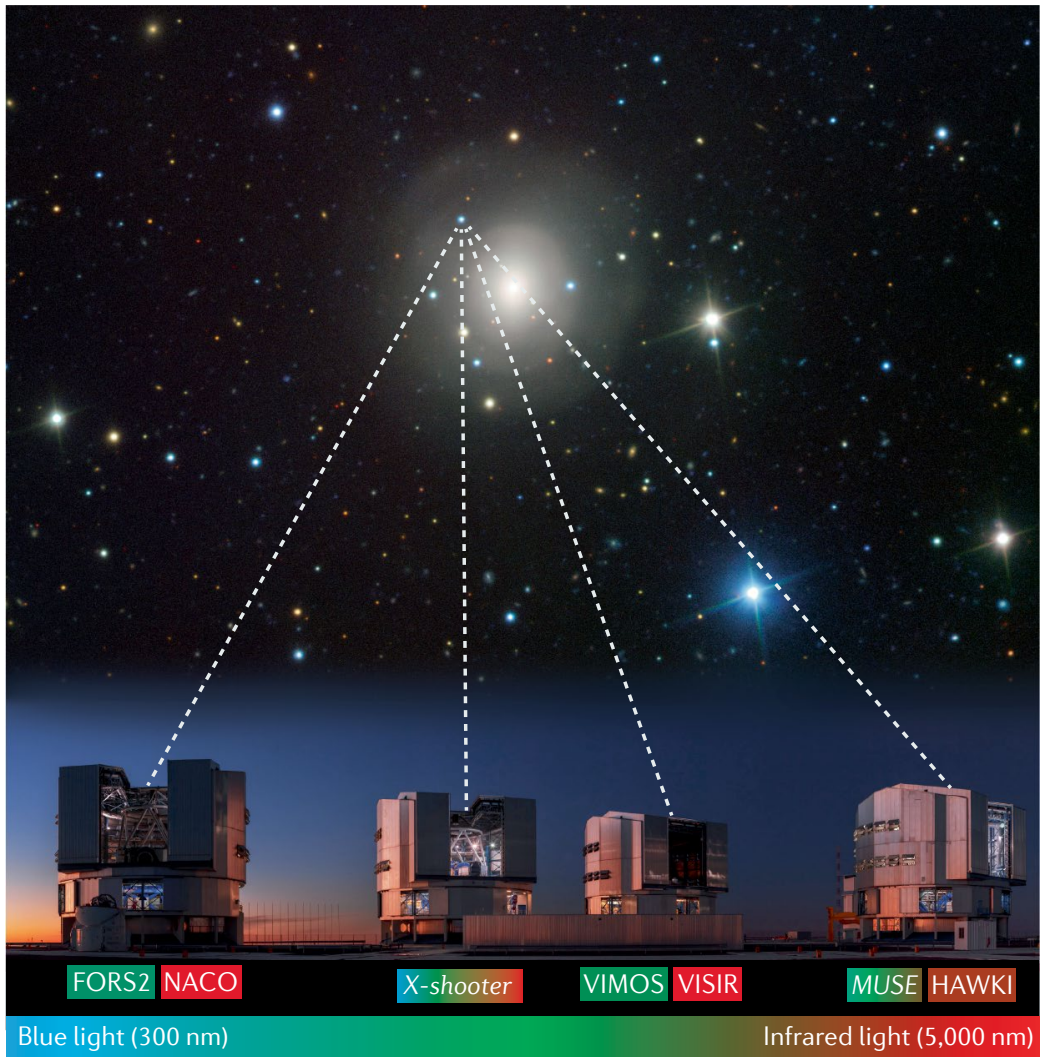

Fig. 1 | The range of facilities at European Southern Observatory (ESO)'s Paranal observatory that made observations (often simultaneously) of GW170817. The simultaneity is vital since the sources can vary quickly, and in the case of GW170817 observations were only possible for an hour each night. The lower bar shows the wavelength range, and the colour of the text boxes corresponds to the wavelength of each individual instrument. X-shooter and the Multi-Unit Spectroscopic Explorer (MUSE) were used predominantly for spectroscopy. The instruments include the FOcal Reducer and low-dispersion Spectrograph (FORS), the Naysmith Adaptive Optics

System Imager and Spectrograph (NACO), the X-shooter spectrograph, the Visible MultiObject Spectrograph (VIMOS), Very Large Telescope (VLT) Imager and Spectrograph for mid-infra-red (VISIR), MUSE and High Acuity Wide Field K-band imager (HAWKI). The top image is a multicolour image from the VIMOS instrument and is adapted courtesy of ESO, A. J. Levan, N. R. Tanvir. The bottom image is reprinted courtesy of ESO/B, Tafreshi (twanight.org).
Of course, creating a consortium doesn't necessarily provide the resources necessary to undertake the research. ENGRAVE has, therefore, had to earn large amounts of precious telescope time. In doing this, we have enlarged from our initial focus on the telescopes at ESO to a wider range of multi-wavelength effort that includes significant time allocations with the Atacama Large Millimetre Array (ALMA) in the Atacama Desert in Chile, facilities on the Canary Islands such as the William Herschel telescope and most notably the Spanish Gran Telescopio Canarias, and the Hubble Space Telescope.

It was with this significant set of resources that ENGRAVE approached the third LIGO/Virgo science run (O3). Whereas GW170817 was a close and probably rare event, it was likely that we would require more effort to identify and study new counterparts. Indeed, over the O3 run, only about four credible neutron star-neutron star or neutron star-black hole mergers were found, all at much larger distances than GW170817. For each of these, our alert system informed collaboration members of the event within a few minutes of its announcement, and the searches swung into action. In the end, no viable counterparts were found in $\mathrm{O} 3$, but there were other highlights.

On 14 August 2019, the LIGO and Virgo detectors identified a likely signal that appeared to arise from the merger of a neutron star with a black hole, a hitherto unseen combination. The source was localized to just a few square degrees (with $50 \%$ confidence), with a distance eight times larger than GW170817. ENGRAVE obtained a series of observations of galaxies within the error box, both directly with the VLT, and in collaboration with a range of wide-field telescopes. We did not identify a counterpart, but set some of the most stringent limits on the presence of a counterpart yet possible ${ }^{5}$; perhaps the black hole completely swallowed the neutron star and no mass was ejected to create electromagnetic emission, or maybe the lower mass object was itself a black hole.

\section{The future}

The COVID-19 situation has prematurely suspended both the $\mathrm{O} 3$ run and the operations at ESO. ENGRAVE is now preparing for the next LIGO/Virgo observing run, where more sensitive detectors should yield more events and also reach to greater distances. Meanwhile, we still have data obtained in the last period for some likely gravitational wave events. Furthermore, the targets that we search for, the kilonovae, can be found in wide-field searches irrespective of the gravitational waves. We are now gearing up for such a search.

Ultimately, ENGRAVE is a new experience for many European astronomers. It mirrors a direction of travel that much of astronomy is taking. In doing so, it acknowledges that big science often requires big teams, and that coordination is needed. Indeed, there are even now efforts to coordinate gravitational wave follow-up on a worldwide scale, with a 'treasure-map' where observers can report their searches and discoveries in real time.

There are many challenges in managing the transition from small to large groups. How do we maintain a sense of belonging, a variety of work, scientific agility and speed? How do we adequately recognize the vital work 
of individuals, and ensure they are recognized externally, through grants or academic positions? Ultimately, the success of this enterprise will be judged by its results. We are quietly confident that we can deliver on our scientific promise given the vast range of skills and expertise at our disposal. In doing so, we must also recognize that our collaboration is built of individuals, and it is perhaps they who will ultimately tell us how well we have done.

1. Mészáros, P. et al. Multi-messenger astrophysics. Nat. Rev. Phys. 1, 585-599 (2019).

2. Pian, E. et al. Spectroscopic identification of r-process nucleosynthesis in a double neutron-star merger. Nature 551, 67-70 (2017)
3. Smartt, S. J. et al. A kilonova as the electromagnetic counterpart to a gravitational-wave source. Nature 551, 75-79 (2017).

4. Covino, S. et al. The unpolarized macronova associated with the gravitational wave event GW 170817. Nat. Astron. 1, 791-794 (2017).

5. Ackley, K. et al. Observational constraints on the optical and near-infrared emission from the neutron star-black hole binary merger S190814bv. Preprint available at: arXiv: 2002.01950 (2020).

\section{Competing interests}

The authors declare no competing interests.

RELATED LINKS

More information about ENGRAVE can be found at: http://www.

engrave-eso.org/ 